\title{
Le français parlé radiophonique : Contact de langues et alternance codique
}

\author{
Sadi, Nabil \\ Maître de conférences en Sciences du langage \\ Laboratoire LAILEMM, Université de Béjaia, Algérie
}

Les définitions de l'alternance codique abondent et la terminologie est variée. Certains auteurs caractérisent le phénomène en tant que processus psycholinguistique, d'autres renvoient au statut linguistique de cette pratique langagière, tandis que d'autres enfin désignent le produit linguistique qui en résulte.

Nous avons en français : alternance de langues, alternances codique, mélange de langues, mélange codique, marques transcodiques, incorporation (Boyd, Anderson et Thornell, 1991), bouée transcodique, structures mixtes (Canut, 2002), etc., et en anglais : code-switching, code-mixing, language alternation, intra-sentential code switching, inter-sentential code switching, extra-sentential code switching, odd switching (Gardner-Chloros, 1991), tag-switching, situationnal switching et metaphorical switching, (Blom \& Gumperz, 1972), conversational switching (Gumperz, 1989b), language mixing (Auer, 1999), emblematic switching (Milroy \& Muysken, 1995), fluent code-switching (ou skilled code-switching) / flagged switching (Poplack, 1988b), etc.

Le terme alternance codique s'inscrit par ailleurs dans l'ensemble plus large des «marques transcodiques », terme référant à «l'ensemble des phénomènes de contact linguistique, tels que codeswitch, mélange de langue, interférence, emprunt, etc.» (Lüdi, 1991: 54). C'est ce qui fait que «lorsqu'on se propose d'étudier l'alternance codique ou tout simplement les phénomènes liés aux contacts des langues, on est confronté à un problème de choix d'angle d'attaque et de frontière. » (Zongo, $2004: 27)$.

Ali-Bencherif (2008 : 47) donne les définitions les plus pertinentes selon l'axe d'analyse et selon la vision de chaque auteur dans sa thèse de doctorat : l'alternance codique, selon lui, est l'usage alternatif de deux codes dans une conversation. Une telle définition peut signifier d'une manière générale et avec beaucoup de réserves, qu'il s'agit de conversations bilingues. L'alternance codique dans la conversation peut se définir avec Gumperz (1989a : 57) comme : « la juxtaposition à l'intérieur d'un même échange verbal de passages où le discours appartient à deux systèmes ou sous-systèmes grammaticaux différents. Le plus souvent l'alternance prend la forme de deux phrases qui se suivent. Comme lorsqu'un locuteur utilise une seconde langue soit pour réitérer son message soit pour répondre à l'affirmation de quelqu'un d'autre ». Ce qui attire l'attention dans cette définition c'est l'aspect linguistique qui caractérise l'échange verbal par la présence des énoncés de deux systèmes différents, là où la juxtaposition et la succession laissent entendre que les locuteurs produisent des énoncés bilingues structurés grammaticalement sans qu'il y ait une rupture au niveau de la forme. Dans ce cas-là, il s'agit d'habitudes verbales acquises ou apprises spécifiques aux sujets parlants bilingues, ce qui renseigne aussi sur l'appropriation partielle ou totale de la grammaire des deux langues ainsi qu'une grammaire commune ayant une fonction régulatrice des échanges, où la qualité des énoncés alternés est prise en compte comme fondamentale assurant la communicabilité et l'interaction. À partir de là, l'accent peut être mis sur le rôle de l'alternance codique dans la régulation du discours du locuteur bilingue ou supposé bilingue. On peut souligner également que cette définition s'inscrit dans une perspective fonctionnelle d'orientation interactionnelle. Elle repose essentiellement sur le fait conversationnel où les locuteurs sont inconscients de leurs choix de langues car l'objectif principal est l'intercompréhension, et c'est pourquoi d'ailleurs Gumperz distingue l'alternance codique conversationnelle et l'alternance codique situationnelle. 
Haugen (1950b : 211), quant à lui, reconnaît l'existence du fait que les bilingues passent d'une langue à l'autre mais précise qu' «à tout instant ils ne parlent qu'une langue, même s'ils font appel à une autre ». Pour cet auteur, le mélange codique ne peut pas exister : "l'introduction d'éléments d'une langue dans une autre langue signifie un passage à l'autre langue, et pas un mélange des deux » (ce qui constituerait une anomalie selon lui).

Le paysage sociolinguistique en Algérie se caractérise par l'existence de plusieurs langues (et variétés de langue) régies par des règles historiques, sociopolitiques et culturelles bien déterminées. «[...] La particularité du marché linguistique d'un pays comme l'Algérie réside dans le fait qu'outre la relation ambiguë qui existe entre les langues nationales, arabe et amazighe, la confrontation s'étend à une langue étrangère, voire à deux ou plus » (Mabrour, 2007 : 12-20).

Dans ce sens, Queffélec souligne que

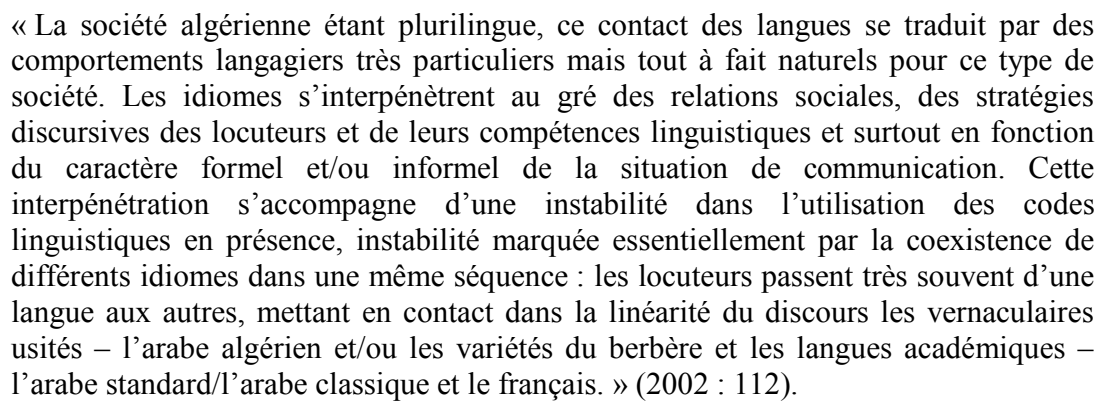

Au cours des différentes interactions, les locuteurs algériens utilisent leurs compétences langagières pour divers besoins de communication. Barillot identifie parmi ces dernières le code switching :
«L'utilisation du langage est le produit de la socialisation de l'individu ; lorsqu'il entre en interaction avec d'autres locuteurs, il use de toutes ses compétences langagières afin de mieux faire passer le message. Parmi ces compétences, on trouve l'utilisation de plusieurs langues dans le même discours : il s'agit du code switching, l'utilisation de deux ou plusieurs langues dans le même discours. » (2002 : 119-134).

Dans notre travail ${ }^{1}$, nous nous confrontons à une situation d'interaction où différents locuteurs construisent un discours riche en alternance codique. Nous y aborderons le français parlé en contact avec d'autres langues, dans une situation de plurilinguisme. Il sera question de la dimension fonctionnelle de l'alternance codique où nous essayerons de déterminer les facteurs du choix et du mélange de langues de la part de différents locuteurs de différentes émissions radiophoniques de la chaîne III, à savoir Le rendezvous de l'économie, Conseils et vous et Micro ondes.

La perspective ${ }^{2}$ interactionniste interroge deux types de problématique : les buts et les motivations des choix de langues et de l'alternance codique multilingue soit dans les communications intergroupes ethnolinguistiques, soit dans les communications intragroupes ethnolinguistiques. Dans cette perspective, l'alternance codique est considérée comme un système autonome: le "vernaculaire bilingue » (Gafaranga, 1997), le « code alterné du bilingue » (Hamers et Blanc, 1883), une «interlangue fossilisée » ou entrelangue (Duran, 1994 ; Baggioni, 1997; Baggioni et Robillard, 1990), un « fused lect» (Auer, 1998), « le parler bilingue » (Py, Lüdi). L'alternance codique est abordée dans une optique interprétative, c'est-à-dire comme une stratégie de communication ${ }^{3}$. Cette perspective vise à décrire les facteurs microsociolinguistiques et macrosociolinguistiques de l'alternance codique (Breitborde, 1983) d'une part (Clyne, 1967 ; Gal, 1979 ; Valdès-Fallis ; 1978 ; Gardner-Chloros, 1983), ses fonctions discursives et conversationnelles liées aux normes sociales et aux relations interpersonnelles d'autre part (Scotton et Ury, 1977 ; Savilles-Troike, 1982 ; Guemperz, 1982, 1989 ; Myers-Scotton 1983 ; Deprez, 1999) (Zongo, $2004: 40)$. 


\section{Le corpus}

L'Algérie dispose de trois chaînes radiophoniques publiques et nationales : la chaine I qui émet ses programmes en arabe, la chaîne II en kabyle et la chaîne III en français, à côté de nombreuses chaînes locales. L'objectif de notre travail étant de faire une analyse d'un discours oral (radiophonique), d'étudier les caractéristiques du français parlé dans un contexte interactionnel et dans une situation de contact de langues, et de déterminer les facteurs qui régissent l'alternance codique, nous avons choisi la chaîne III afin de disposer d'un discours en langue française. Le choix des émissions sur lesquelles nous avons travaillé repose sur des critères que nous avions délimités au départ et qui correspondent aux caractéristiques de notre sujet, telles que les particularités des thèmes des émissions et des interactants.

Nous proposons de présenter en premier lieu Le rendez-vous de l'économie (5 numéros), une émission qui traite de plusieurs aspects de l'économie, qu'elle soit nationale ou internationale, tels que les activités industrielles, financières et commerciales du monde en général et de l'Algérie en particulier. En deuxième lieu, nous avons choisi Conseils et vous (4 numéros), une émission qui représente un espace d'expression pour les gens en détresse, ayant des problèmes de cœur, des problèmes professionnels, personnels, familiaux, etc. En dernier lieu, nous nous sommes intéressé à Micro ondes (5 numéros), une émission placée sous le signe de la musique de tous genres, de divertissement, de jeu et de plaisir. La transcription ${ }^{4}$ des enregistrements (quatorze numéros) a donné un total de 255 pages, soit 104284 mots. 40293 mots sur 79 pages dans Le rendez-vous de l'économie, 30369 mots sur 71 pages dans Conseils et vous et enfin 33622 mots sur 105 pages dans Micro ondes.

Chaque fois qu'un locuteur choisit de parler une langue et par conséquent chaque fois qu'il change de langue, ses motivations peuvent être multiples. Cette alternance n'est pas le fruit du hasard mais elle est régie par plusieurs facteurs que nous pouvons dégager à travers l'analyse de notre corpus. En effet, l'observation des énoncés produits pas les différents locuteurs des trois émissions fait ressortir deux groupes de facteurs qui représentent les différentes raisons qui ont poussé les locuteurs à faire de l'alternance.

\section{Les facteurs liés aux caractéristiques du langage parlé}

«Toute conversation spontanée paraît, de premier abord, désordonnée et désarticulée dans une description minutieuse mais, en fait, elle est ordonnée et logique. Seulement, on ne peut l'ordonner selon la même façon que le texte écrit où la grammaire règne sans partage » (Manaa, 2000). Le langage parlé trouve sa cohérence dans plusieurs mécanismes différents comme la répétition de mots ou de propositions, les phrases courtes, les pauses et les interruptions qui lui donnent un aspect de discontinuité supplémentaire.

\subsection{Le déclenchement}

Le déclenchement est un phénomène qui caractérise le langage parlé et qui peut expliquer le changement de langue. Clyne (1967) part de l'hypothèse que ce changement est motivé par un phénomène de « trigging », c'est-à-dire de déclenchement du code switching par un élément linguistique déjà produit par le locuteur ou l'interlocuteur. «Ce qu'on dit est souvent déterminé par un énoncé antérieur ou par une anticipation par rapport à ce qui suit. Tout transfert (marque transcodique) peut conduire l'esprit du locuteur à la langue qui est source de transfert et peut donc causer d'autres exemples de transferts morphologiques ou morphosyntaxiques et phoniques aussi bien que multiples » (Clyne, 1967: 84, cité par Zongo, 2004).

Nous avons recensé quelques extraits ${ }^{5}$ qui témoignent de ce phénomène où un locuteur est influencé par un mot ou une expression dans la langue qu'utilise son interlocuteur :

$\mathrm{I}_{3}:$ je voudrais répondre à Si (monsieur) Hakim/ pour lui dire deux choses $\uparrow$ L. 405

[...] L. 416 


\begin{abstract}
$\mathrm{I}_{2}$ : merci monsieur Hamid// moi je voudrais juste euh euh répondre un petit peu euh enfin répondre euh rebondir sur ce qu'a déclaré mon ami $\mathrm{Si}$ (monsieur) Abderrahmane (E. 1, N.1, P. 11, L.405-421)
\end{abstract}

Comme nous pouvons le constater, le deuxième invité passe du français à l'arabe, en désignant son ami par Si (monsieur). Il a été influencé par le troisième invité qui a utilisé la même expression en répondant à un auditeur (Hakim). Plus loin dans la conversation, l'animateur reprend également l'expression en s'adressant à un autre auditeur (Khaled) interpellé auparavant par le même invité :

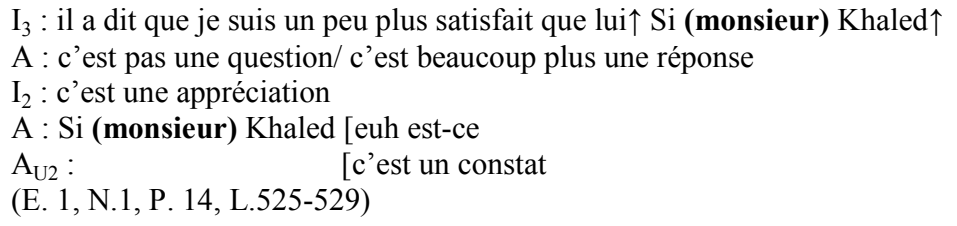

Il s'agit dans ce cas d'emprunt et non pas d'une instance d'alternance codique, dans la mesure où les locuteurs insèrent un mot arabe dans un énoncé en français.

Weinreich, dans son ouvrage fondateur de 1953, Languages in contact : findings and problems, adopte un point de vue similaire. S'il reconnaît, comme Haugen, que « le bilingue passe d'une langue à l'autre en fonction de changements appropriés dans la situation de communication interlocuteurs, thèmes, etc.) », il nie par contre la possibilité qu'un tel phénomène apparaisse à l'intérieur d'une phrase (Weinreich, 1953 : 73). Quant à la présence d'éléments lexicaux d'une langue apparaissant dans des énoncés d'une autre langue, il ne peut s'agir selon lui que d'emprunts.

D'autres exemples de déclenchement sont à retenir où un mot ou une expression dans une langue provoque une chaîne de mots dans cette langue. En attestent les extraits suivants tirés de la deuxième émission (Conseils et vous) :

$\mathrm{A}_{\mathrm{U} 1}$ : oui/ bonsoir Sabrina

A : bonsoir Djamila

$\mathrm{A}_{\mathrm{U} 1}$ : vous allez bien ?

A : elhamdoullah (louange à Dieu)

$\mathrm{A}_{\mathrm{U} 1}$ : la famille va bien?

A : ça va

$\mathrm{A}_{\mathrm{U} 1}$ : toute l'équipe va bien?

A : tout le monde se porte bien et [tout le monde est ravi de vous avoir au bout du fil//

$\mathrm{A}_{\mathrm{U1}}$ : [elhamdoullah (louange à Dieu)// et ben moi aussi je

suis ravie euh je voulais vous appeler bien avant ce jour-là mais malheureusement

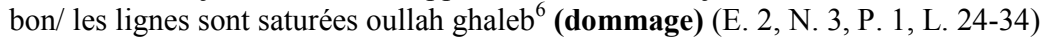

$\mathrm{A}_{\mathrm{U} 2}$ : ça va Sabrina?

$\mathrm{A}$ : ça va je vous remercie (rires) et vous?

$\mathrm{A}_{\mathrm{U} 2}$ : ça va

A : elhamdoullah (louange à Dieu)

$\mathrm{A}_{\mathrm{U} 2}$ : allah yselmek (que Dieu te protège)

A : ça va bien?

$\mathrm{A}_{\mathrm{U} 2}$ : ça va très bien elhamdoullah (louange à Dieu)/ je vous embrasse très fort (E. 2,

N. 3, P. 4, L. 116-122)

Ces deux extraits représentent des conversations entre l'animatrice et les auditeurs. En prenant l'appel, ces derniers saluent l'animatrice en lui demandant comment elle va en utilisant le français. L'animatrice leur répond en arabe, ce qui les fait réagir dans la même langue en reprenant ses dires et en alternant les deux langues à travers des formules d'invocation à Dieu et des expressions votives. 
En effet, dans le premier exemple, l'auditrice reprend elhamdoullah (expression utilisée auparavant par l'animatrice pour dire qu'elle allait bien) en sachant que toute l'équipe se portait bien, et alterne les deux langues en exprimant son mécontentement de ne pas pouvoir les appeler souvent.

Le même phénomène apparaît chez la deuxième auditrice qui passe du français à l'arabe, d'un tour de parole à un autre. Elle enchaîne en alternant le français et l'arabe et en utilisant la même expression, pour enfin conclure en français.

L'énoncé suivant regorge d'exemples de déclenchement. Les locuteurs utilisent simultanément les mêmes expressions.
A : alors Meriem/ du nouveau ?/ votre mari est revenu de voyage ?
$\mathrm{A}_{\mathrm{U} 2}$ : oui/ mais je suis vraiment triste parce que euh je viens de rentrer à la maison/ soudain j'ai entendu Djamila qui euh qui me dit que madame Hassina est décédée ellah yerhamha $\downarrow$ (que Dieu lui accorde sa miséricorde)
A : ellah yerhamha inchallah $\downarrow$ (que Dieu lui accorde sa miséricorde s'il le veut)
$A_{\mathrm{U} 2}$ : inchallah (si Dieu le veut)/ elle nous laisse un euh un grand vide/ rebbi yerhamha ouyouesaa aaliha $\downarrow$ (que le Seigneur lui accorde sa miséricorde et l'accueille en son vaste paradis)
A : inchallah (si Dieu le veut)
$\mathrm{A}_{\mathrm{U} 2}$ : je voudrais vraiment euh// dire à ses enfants mes sincères condoléances/
A : elle n'a pas d'enfants hein/ L. 135[...] L. 140 elle nous a rappelé pour nous dire que son mari était décédé ellah yrahmou $\downarrow$ (que Dieu lui accorde sa miséricorde)
$\mathrm{A}_{\mathrm{U} 2}$ : ellah yrahmou $\downarrow$ (que Dieu lui accorde sa miséricorde) $\quad$ (E. 2, N. 3, P. 4, L. 125-142)

En parlant en français d'une femme décédée, l'auditrice prie Dieu de lui accorder sa miséricorde en utilisant l'arabe. L'animatrice fait de même en ajoutant l'expression inchallah, laquelle est reprise par l'auditrice avant qu'elle enchaîne en français. Elle prie en arabe une deuxième fois en demandant à Dieu de l'accueillir en son vaste paradis, d'où le rebondissement en arabe de l'auditrice. Cette dernière prie aussi pour son mari décédé en passant du français à l'arabe avec la même expression, reprise juste après par l'auditrice.

Nous constatons que les expressions relatives à la religion, exprimées en arabe dans un discours en français, sont aussitôt reprises par les locuteurs dès qu'elles sont employées par leurs interlocuteurs. Ces expressions peuvent être dites en français mais les locuteurs choisissent l'arabe pour exprimer leur appartenance religieuse et culturelle. Il s'agit des vœux et des formules de prières suite à un décès, que nous aborderons dans les facteurs liés à la religion. En voici d'autres exemples pour l'utilisation de l'expression votive inchallah:

A : j'espère que ça sera le cas pour vous/ et que vous nous appellerez dans quelques années pour nous dire la même chose/ nous vous souhaitons votre trentième anniversaire de mariage inchallah (si Dieu le veut) [ça nous fera plaisir

AU2 :

(E. 2, N. 3, P. 6, L. 183-186)

[inchallah (si Dieu le veut)/ ah oui je le sais

A : écoutez on vous embrasse aussi de notre côté Meriem/ et puis encore une fois passez de très très belles vacances

AU2 : inchallah (si Dieu le veut) merci/ je suis vraiment désolée mais/ l'heure de l'émission de la chanson africaine?

A : alors ce sera euh pour cette semaine euh mercredi après le journal africain dix-huit heures quinze inchallah (si Dieu le veut) et puis je vous donnerai les horaires ce jourlà/ les horaires de l'été//

AU2 : inchallah (si Dieu le veut)

(E. 2, N. 3, P. 8, L. 248-255) 


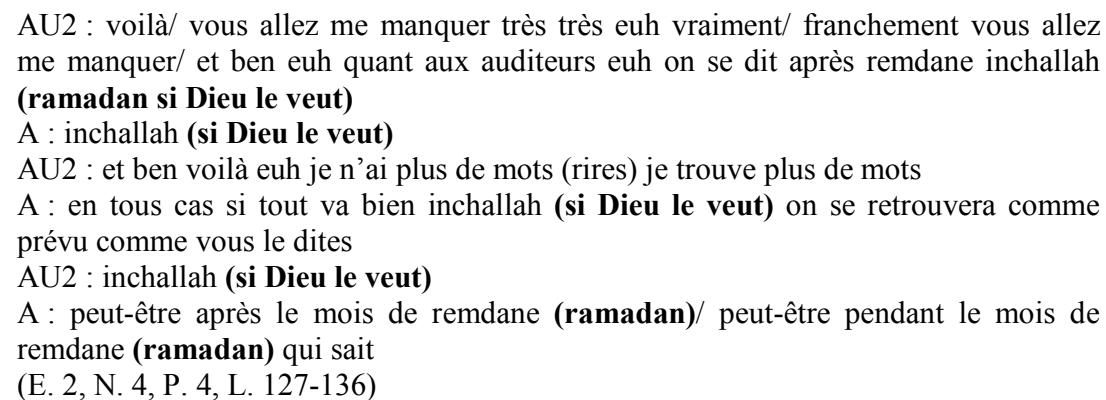

Dans le dernier passage, l'animatrice est influencée par l'auditrice, qui fait référence au mois de carême, en passant du français à l'arabe. Au début de la conversation, elle reprend inchallah utilisé par l'auditrice qui lui donne rendez-vous après remdane, qu'elle reprend à deux reprises plus loin dans la conversation.

Contrairement aux deux premières émissions, le phénomène de déclenchement dans Micro ondes ne se résume pas à la reprise d'éléments produits dans une langue autre que la langue de base. Les différents locuteurs alternent deux langues dès que les autres le font, à savoir soit l'arabe et le français, soit le français et l'anglais. Les auditeurs changent souvent de langue d'un tour de parole à un autre en étant influencés par l'animateur. En voici quelques extraits :

A : dis-moi $\uparrow /$ les Algérois conduisent toujours aussi bien?

$\mathrm{A}_{\mathrm{U} 5}$ : euh ça va ça s'améliore avec les travaux euh avec tout ce qui est en train de se faire/ normalement ça va s'améliorer

A : kima qal ouahed $\uparrow$ (comme quelqu'un a dit)// elle est bien goudronnée

$\mathrm{A}_{\mathrm{U} 5}$ : zaâma $\uparrow$ (soi-disant) soi-disant

(E. 3, N. 1, P. 6, L. 190-194)

A : vingt-deux voix $\uparrow /$ et toi aussi tu voudrais savoir ce que c'est que l'amour?

$\mathrm{A}_{\mathrm{U} 7}:$ pardon $\uparrow$

A : toi aussi tu voudrais savoir ce que c'est que l'amour ?/ you want to know what love is?

$\mathrm{A}_{\mathrm{U} 7}$ : yes no

(E. 3, N. 1, P. 10, L. 294-297)

A : alors o $\mathrm{p} \uparrow \mathrm{o} \mathrm{p}^{7} \uparrow / /$ non/ pas d'idée// entre temps/ vous donnez une voix à qui Mohamed?

$\mathrm{A}_{\mathrm{U} 1}$ : euh Foreigner

A: Foreigner $\uparrow$ with «I Want To Know What Love Is »/ [trente-trois voix

$\mathrm{A}_{\mathrm{U} 1}:[$ yes

(E.3, N.5, P. 5, L. 157-160)

$\mathrm{A}_{\mathrm{U} 10}$ : et viens à Oran/ on t'invite à Oran

$\mathrm{A}:$ ah $\uparrow$ tdirouri (vous me ferez) du bon poisson

$\mathrm{A}_{\mathrm{U} 10}$ : arouah neddik (viens je t'emmènerai) à la pêcherie

(E. 3, N. 5, P. 20, L. 619-621)

$\mathrm{A}_{\mathrm{U10}}$ : et aussi Karim ouldi (mon fils)/ j'ai pas reçu de puce $\uparrow /$ j'ai gagné trois puces/ ourahoum euh ou gheblouni (elles sont euh ils m'ont dupée)

A : choufi choufi (regarde regarde)/ pour nous excuser

$\mathrm{A}_{\mathrm{U} 10}$ : ouah (oui)

(E. 3, N. 5, P. 20, L. 604-607)

Dans les trois premiers extraits, les auditeurs sont influencés par la langue qu'utilise l'animateur, dans la mesure où ils passent d'une langue à une autre d'un tour de parole à un autre. En effet, dans le premier passage, l'auditeur répond aussi en français à la question de l'animateur, mais dès que ce dernier alterne l'arabe et le français, il le suit aussitôt dans sa pratique. Le même phénomène apparaît dans le deuxième 
et le troisième passage, où les auditeurs rebondissent en anglais. L'auditeur $\left(\mathrm{A}_{\mathrm{U}}\right)$ répond à la question posée en français, puis reformulée en anglais par l'animateur, tandis que l'auditeur $\left(\mathrm{A}_{\mathrm{U} 1}\right)$ confirme par yes le classement annoncé par l'animateur.

Dans les deux derniers passages, la conversation se tient entre l'animateur et le même auditeur $\left(A_{U 10}\right)$. Nous remarquons une influence mutuelle entre les deux locuteurs, dans la mesure où l'un deux alterne l'arabe et le français dès que l'autre le fait.

\title{
2.2 Le besoin lexical
}

L'alternance peut témoigner également d'une incompétence linguistique, lacunaire ou insuffisante où le locuteur fait appel à une autre langue afin de répondre à un phénomène de domination linguistique ou à des trous de mémoire. Nous avons sélectionné quelques exemples où le changement de langue semble se produire à n'importe quelle jonction du discours :

\begin{abstract}
$A_{U 2}$ : j'ai envie d'éclairer ceux qui écoutent/ je suis des travaux publics/ vos invités me connaissent/ alors ou vous me laissez dire ce que j'ai à dire entièrement oulla ma neqbelch (si non je refuse) L. 460 [...] L. 475 et enfin un dernier point/ el moqarana bine (la comparaison entre) les routes en bitume et les routes en béton (E. 1, N. 3, P. 12-13, L. 458-476)

$\mathrm{A}_{\mathrm{U} 1}$ : euh ça va très bien mais euh seulement c'est la dernière dernière/ ça fait un peu mal/ cette émission va nous manquer/ L. 27[...] L. 31 franchement c'est à ça seulement euh laâbad berk elli (les gens seulement qui) euh qui appellent pour nous dire pour nous encourager// pour ces petits conseils qu'on/ qu'on communique (E. 2, N.4, P.1-2, L. 26-33)

$A_{U 1}$ : elle va nous manquer cette émission// et en même temps je voudrais seulement dire aux auditeurs de euh el insane elli yeqder yerfaâ yeddih l'sma ou yqoul/ ellah yrahmek ya Hassina (l'être humain qui peut lever ses mains vers le ciel et dire que Dieu t'accorde sa miséricorde Hassina)// rebbi yerhamha ou yadjaal trabha hnine ou inchallah yaâtiha khir m'denya ou khayrane f'el akhira (que le Seigneur lui accorde sa miséricorde et lui rende la terre douce et si Dieu le veut, qu'il lui accorde dans l'au-delà plus et plus et mieux que dans la vie ici-bas)

(E. 2, N.4, P.1-2, L. 34-40)
\end{abstract}

Dans les deux premiers passages, les locuteurs combinent l'arabe et le français en introduisant des segments en arabe dans un discours à base française. Les locuteurs initient leur énoncé en français, passent à l'arabe puis reviennent au français pour conclure. Ce choix s'explique soit par le besoin des locuteurs de faire passer un message à travers des expressions appropriées, soit pour ne pas rompre la conversation en choisissant l'arabe comme langue du maintien du discours. Dans le premier passage " oulla ma neqbelch » dénote l'insistance du locuteur et son caractère catégorique (énervement). Ce choix peut s'expliquer aussi par des lacunes de vocabulaire où les segments passent mieux en arabe qu'en français, tels que « el moqarana bine » (exemple 1) et « laâbad berk elli » (exemple 2).

Dans le dernier extrait, l'auditrice passe du français à l'arabe pour conclure en priant Dieu pour une femme décédée. Tout le passage qui véhicule un sentiment de tristesse et de prière est exprimé en arabe, la langue de la religion musulmane.

D’autres exemples témoignent également de ce recours à l'arabe en faisant référence à Dieu : 


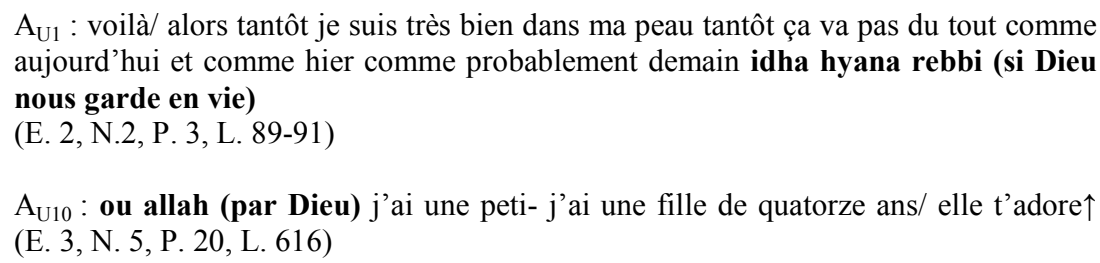

Dans le premier passage, l'auditrice passe du français à l'arabe pour prier Dieu de la garder en vie, tandis que dans le deuxième exemple, l'auditeur appuie en arabe son affirmation en utilisant la formule de serment " ou allah » et enchaîne en français.

Cette alternance peut s'expliquer également par des trous de mémoire, où l'insertion de comment dirais-je renvoie à l'hésitation du locuteur et indique la recherche de mots comme cela apparaît dans l'extrait suivant :

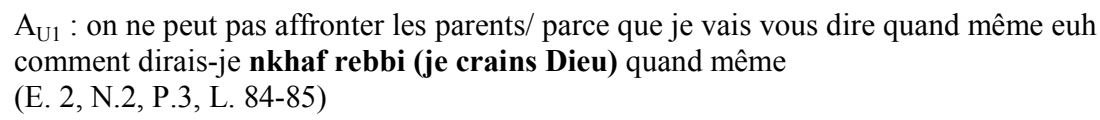

\subsection{Les marqueurs de discours}

Les locuteurs utilisent divers éléments linguistiques dans leurs discours afin de donner à leurs conversations plus de fluidité :

$\mathrm{A}_{\mathrm{U} 1}$ : et là je voudrais euh je voudrais tant que ma participation apporte yaâni (ça veut dire) un euh un bénéfice pour euh toute la famille algérienne// euh voilà moi je suis issue d'une famille de quatre sœurs et un frère

(E. 2, N.2, P.1, L. 31-33)

$A_{U 1}$ : je leur dis un mot mal placé oula (ou) ils vont mal prendre la chose donc je préfère tout prendre sur moi et euh c'est comme ça $\uparrow$ (E. 2, N.2, P.3, L. 86-87)

$\mathrm{A}_{\mathrm{U10}}$ : et aussi Karim ouldi (mon fils)/ j'ai pas reçu de puce $\uparrow / \mathrm{j}$ 'ai gagné trois puces/ (E. 3, N. 5, P. 20. L. 604)

Nous constatons à travers ces extraits que les locuteurs enchaînent en insérant des lexies en arabe à l'intérieur de leur discours à base française. Ainsi, "yaâni », " oula » et "ouldi » permettent aux auditeurs de s'exprimer aisément sans interrompre leur discours, ce qui témoigne de leur capacité à utiliser les deux codes.

\subsection{Les habitudes et/ou tics de langage (les salutations / les votifs)}

Il est frappant de souligner que des expressions qui ont une valeur symbolique dans les pratiques langagières des locuteurs algériens font l'objet d'alternance dans notre corpus. Les différents locuteurs se saluent, prient, expriment des vœux et invoquent Dieu en arabe. Il s'agit d'insertions, à l'intérieur d'un discours en français, d'éléments relatifs à la culture et à la religion musulmane. Cette pratique est observée dans les trois émissions, avec une prédominance dans Conseils et vous où les conversations se focalisent sur les problèmes sociaux ainsi que sur la vie personnelle des auditeurs, rendant ainsi les conversations riches en émotions.

En ce qui concerne les conversations entre les auditeurs et les animateurs, elles débutent toujours en français. Les locuteurs se saluent en français, s'échangent des expressions de bienvenue puis répondent avec une formule votive. En voici quelques exemples : 
$\mathrm{A}$ : très bien $\uparrow /$ zéro vingt et un quarante-huit quinze quinze/ un appel euh monsieur Hakim d'Alger/ bonsoir Hakim

$\mathrm{A}_{\mathrm{U} 1}$ : bonsoir monsieur Hamid

A : ahlane $\uparrow$ mrahba (bienvenue)

(E. 1, N. 1, P. 10, L. 372-375)

A : donc on prend l'appel au zéro vingt et un quarante-huit quinze quinze/ Khaled $\uparrow$ de Mostaganem $\uparrow /$ bonsoir Khaled $\uparrow$

$\mathrm{A}_{\mathrm{U} 2}$ : oui/ bonjour messieurs

A : oui bonjour $\uparrow /$ votre question s'il vous plait $\uparrow /$ mrahba bik (bienvenue à toi)

(E. 1, N. 1, P. 14, L. 504-507)

A : comment ça va Lilya?

$\mathrm{A}_{\mathrm{U} 5}$ : je suis très heureuse de vous contacter pour la première fois

$\mathrm{A}:$ ah $\uparrow$ et bien mrahba bik (bienvenue à toi)

(E. 2, N. 4, P. 11, L. 370-372)

Comme le montrent ces passages, les animateurs souhaitent parfois la bienvenue aux auditeurs avec la même expression en arabe « mrahba bik». Ils prennent à chaque fois l'appel en utilisant le français pour ensuite passer à l'arabe pour les salutations.

$\mathrm{A}_{\mathrm{U} 1}$ : bonsoir monsieur Hamid

A : ahlane $\uparrow$ mrahba (bienvenue)

$\mathrm{A}_{\mathrm{U} 1}$ : ouach rakoum ? labas ? (comment allez-vous ? ça va ?)

A : labas lhamdoullah (ça va, louange à Dieu) et vous-même?

(E. 1, N. 1, P. 10, L. 374-377)

L'animateur souhaite la bienvenue à l'auditeur en arabe après que celui-ci l'a salué en français. Influencé par l'animateur, l'auditeur passe à l'arabe pour lui demander comment il va, celui-ci lui répond en arabe en reprenant «labas » et en enchaînant avec l'expression d'invocation à Dieu « lhamdoullah ».

La même expression caractérise les réponses de l'animatrice de la deuxième émission. Les échangent se déroulent en français jusqu'à ce que l'animatrice utilise « elhamdoullah ».

$\mathrm{A}_{\mathrm{U} 2}$ : bonsoir $\uparrow$

$\mathrm{A}:$ bienvenue à vous $\uparrow$

$\mathrm{A}_{\mathrm{U} 2}$ : ça va bien?

A : ça va $\uparrow$ elhamdoullah (louange à Dieu)

$\mathrm{A}_{\mathrm{U} 2}$ : euh ça fait pas longtemps que j'ai découvert l'émission $\uparrow /$ euh c'est la première

fois que $\mathrm{j}$ 'appelle et euh je vous ai et que je vous ai eu $\uparrow$

(E. 2, N. 1, P. 2, L. 61-66)

A : bonsoir Fatma Zohra

$\mathrm{A}_{\mathrm{U} 3}$ : bonsoir/ ça va bien ?

A : comment ça va?

$\mathrm{A}_{\mathrm{U} 3}$ : ça va merci/ et vous?

$\mathrm{A}$ : alhamdoullah $\uparrow$ (louange à Dieu)/ soyez la bienvenue

$\mathrm{A}_{\mathrm{U} 3}$ : merci

(E. 2, N. 1, P. 6, L. 215-220)

Comme nous pouvons le remarquer, les salutations se font en français ainsi que les questions relatives au bien-être des locuteurs. L'animatrice répond toujours avec la même expression en arabe, en alternance avec le français. Puis la conversation se poursuit en français.

Pour ce qui est de la troisième émission, toutes les salutations et les prises de contact se déroulent en français, sauf avec une auditrice qui utilise également la même expression précédée de «ça va », pour ensuite enchaîner plus loin en français. 


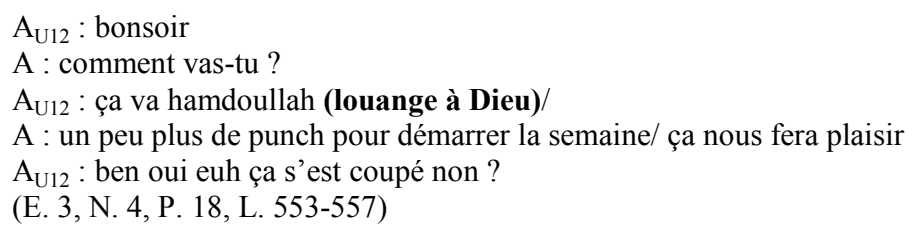

Les expressions relatives aux vœux et aux prières sont également insérées dans le discours à base française. Nous retrouvons des formules qui s'avèrent dans le corpus quantitativement les plus répandues, à savoir «allah ibarek» (littéralement «que Dieu perdure... ») et "inchallah» (si Dieu le veut). La première expression s'utilise avec tout ce qui est quantifiant, que ce soit l'âge, la durée, le nombre d'enfants, etc. La deuxième semble répondre à un besoin d'expressivité.

$\mathrm{A}_{\mathrm{U} 2}$ : euh je suis marié/ ça fait neuf ans que je suis marié

A : allah ibarek (que Dieu te bénisse)

(E. 2, N. 1, P. 2, L. 72-73)

A : vous avez quel âge ?

$\mathrm{A}_{\mathrm{U} 3}: \mathrm{j}$ 'ai trente-trois ans

A : trente-trois ans $\uparrow$

$\mathrm{A}_{\mathrm{U} 3}$ : oui

A : allah ibarek (que Dieu te bénisse)

$A_{U 3}$ : ibarek fik (qu'il te bénisse)

(E. 2, N. 1, P. 10, L. 329-334)

A : il a quel âge?

AU1 : il a douze ans

A : allah ibarek (que Dieu le bénisse)

AU1 : ibarek fik iselmek (que Dieu te bénisse et te protège)

(E. 2, N. 2, P. 3, L. 100-104)

Dans le premier extrait, l'auditrice annonce qu'elle est mariée depuis neuf ans, d'où l'utilisation de " allah ibarek » par l'animatrice.

Par ailleurs, la même expression est utilisée comme réplique à l'âge annoncé par les auditrices. L'animatrice souhaite une longue vie à la troisième auditrice qui a trente-trois ans et au fils de la première qui a douze ans. Les auditrices répondent en utilisant la même expression reformulée au passif ${ }^{8}$, souhaitant ainsi à l'animatrice une longue vie et une paix durable.

Nous avons sélectionné d'autres extraits qui illustrent l'utilisation fréquente et variée de la formule votive "inchallah ».

A : oui $\uparrow /$ et maintenant vous êtes unis pour la vie inchallah (si Dieu le veut)

(E. 2, N. 1, P. 4, L. 126)

A : je vous souhaite encore euh beaucoup beaucoup de bonheur inchallah $\uparrow($ si Dieu le veut) à tous les deux

(E. 2, N. 1, P. 6, L. 182-183)

A : je vous remercie euh je ne sais pas comment vous le dire parce que c'est vrai c'est euh ce sont des mots/ qui euh qui vont peut-être aider les autres à euh à remonter la pente à avoir envie de euh de reprendre goût à la vie inchallah (si Dieu le veut) (E. 2, N. 2, P. 11, L. 375-378)

A : c'est signé Habib $\uparrow$ qui remplace exceptionnellement monsieur Balak/ qu'on retrouve euh très bientôt euh inchallah $\uparrow($ si Dieu le veut)/

(E. 2, N. 1, P. 6, L. 209-211) 


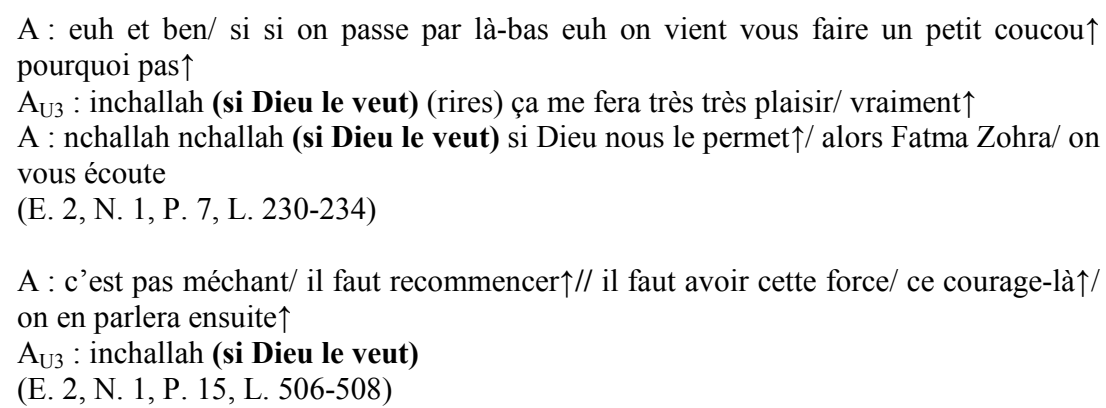

Dans ces six premiers passages la formule votive est utilisée après avoir formulé un vœu, un souhait ou une éventualité. En effet, l'animatrice souhaite une union pour la vie et beaucoup de bonheur à un auditeur (extrait 1 et 2). Elle souhaite également que les gens reprennent goût à la vie en écoutant les conseils des participants à l'émission (extrait 3).

Les locuteurs utilisent aussi cette expression lorsqu'ils évoquent un fait qui se déroulera ou qui peut se produire dans le futur. C'est le cas dans le quatrième extrait où l'animatrice parle du retour de monsieur Balak, et de la possibilité de rendre visite à une auditrice, dans le cinquième extrait. Dans le dernier extrait, il y a une notion de souhait (je te souhaite de bien démarrer la semaine/de recommencer) véhiculée par l'animatrice et à laquelle l'auditrice réplique par « inchallah».

Parfois, l'insertion de ces éléments répond aussi à des besoins d'expressivité que ressentent les auditeurs, tel que l'espoir :

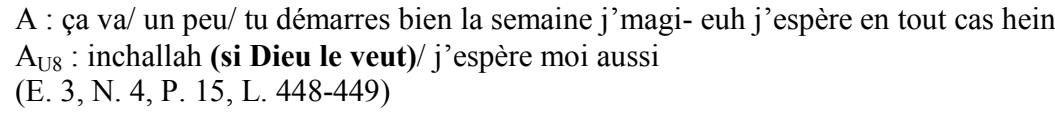

Nous avons noté également que cette expression sert d'invocation à Dieu après une prière. Les locuteurs finissent leurs formules de prière avec « inchallah », pour que Dieu puisse les exaucer.

$\mathrm{A}_{\mathrm{U} 2}$ : oui/ mais je suis vraiment triste parce que euh je viens de rentrer à la maison/ soudain j'ai entendu Djamila qui euh qui me dit que madame Hassina est décédée ellah yerhamha $\downarrow$ (que Dieu lui accorde sa miséricorde) A : ellah yerhamha inchallah $\downarrow$ (que Dieu lui accorde sa miséricorde s'il le veut) $A_{\mathrm{U} 2}$ : inchallah (si Dieu le veut)/ elle nous laisse un euh un grand vide/ rebbi yerhamha ouyouesaa aaliha $\downarrow$ (que le Seigneur lui accorde sa miséricorde et l'accueille en son vaste paradis)

A : inchallah (si Dieu le veut)

(E. 2, N. 3, P. 4, L. 126-133)

Dans ce passage, l'auditrice évoque le décès de madame Hassina et prie Dieu de lui accorder sa miséricorde. L'animatrice formule la même prière en ajoutant « inchallah », repris aussitôt par l'auditrice qui prie Dieu de l'accueillir en son vaste paradis. Ce qui pousse l'animatrice à rebondir avec "inchallah ».

\section{Les facteurs liés aux aspects sémantiques du discours}

Il s'agit ici du choix de la langue qui n'est déterminé ni par la compétence du locuteur dans les deux langues ni par les caractéristiques perçues des interlocuteurs (titres de chansons, habitudes du langage). Ainsi l'alternance permettrait au locuteur d'exprimer leur appartenance culturelle. 


\subsection{Les implicites culturels}

Chaque langue véhicule une culture propre et influence autant qu'elle est influencée par les langues avec lesquelles elle est en contact. Souvent, les participants de l'interaction souhaitent dire d'une manière implicite autre chose que le sens littéral, à travers les insinuations, l'ironie et la métaphore.

Dans les exemples suivants, les locuteurs emploient des expressions figées relatives à la culture algérienne. Ces expressions que nous appelons « implicites culturels » ne véhiculent un sens connoté que lorsqu'elles sont exprimées en arabe dialectal et que seuls les locuteurs partageant cette culture et connaissant cette langue pourraient comprendre.

$A_{U 1}$ : le garçon euh kima y koulou aâla raso richa (comme on dit : sur sa tête une plume) et quatre sœurs/

(E. 2, N. 2, P. 2, L. 38)

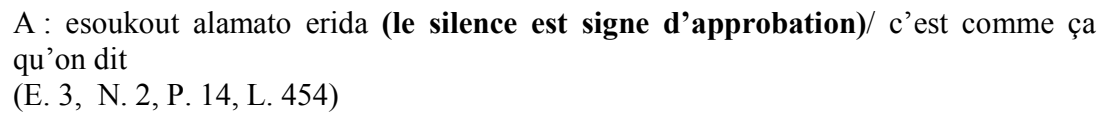

A : un petit coucou également à ma cousine/ qui demain convolera en justes noces// belle comme le jour// elle s'appelle Chinèse Belyout/ très belle/ vingt-quatre ans déjà ::// laâkouba likoum elli qaâdou (bientôt votre tour, vous qui restez)

(E. 3, N. 5, P. 18, L. 555-557)

Dans le premier passage, l'auditeur utilise une expression figée véhiculant un sens connoté que seul un interlocuteur algérien pourrait comprendre. Il précise d'ailleurs que c'est ce qui se dit par tout le monde, à savoir les Algériens, à travers « kima y koulou » (comme on dit).

L'animateur de la troisième émission fait de même en relatant deux expressions ayant une valeur symbolique dans les pratiques langagières des locuteurs algériens. Le proverbe en arabe classique dans le deuxième passage se dit lorsqu'une personne s'abstient de répondre à une question, et cela dénote son approbation, tandis que l'expression «laâkouba likoum elli quaâdou» renferme une valeur expressive assez importante dans la société algérienne lorsqu'on parle de mariage, dans la mesure où on l'utilise pour souhaiter le même bonheur aux célibataires.

\subsection{Les moments de la prière}

Un autre facteur semble régir l'alternance de l'arabe et du français, à savoir les moments de la prière. Au cours des deux premières émissions, les animateurs annoncent l'heure de la prière (prière du début d'après-midi). Que ce soit à la télévision ou à la radio, on interrompt les programmes au moment de l'appel à la prière (el adhane). Les animateurs annoncent toujours ce moment en arabe :

A : très bien// on marque une petite pause euh pour euh annoncer l'heure de la prière d'el dohr (la prière du début d'après-midi)

(E. 1, N. 2, P. 4, L. 242-243)

A : si je dois euh décrypter ce que vous veniez de dire après euh juste avant el adhane (l'appel à la prière)

(E. 1, N. 2, P. 7, L. 247-248)

Exceptionnellement, le deuxième numéro de la première émission Le rendez-vous de l'économie a été diffusé en début d'après-midi, d'où l'annonce de l'heure de la prière d' "el dohr », dans le premier passage. Dans le deuxième extrait, l'animateur revient sur ce dont l'invité parlait avant l'appel à la prière, il passe du français à l'arabe en mentionnant ce moment-là. 
L'animatrice de la deuxième émission fait de même en annonçant l'appel à la prière du coucher du soleil (el maghrib).

A : la suite du programme après l'appel à la prière d'el maghrib $\downarrow$ ( du coucher du soleil) allah yekbel (que Dieu accepte )

(E. 2, N. 1, P. 2, L. 56-57)

A : Linda je voulais juste euh m'excuser auprès de vous puisque euh on va faire une petite parenthèse il y aura el adhane adhane el maghreb (l'appel à la prière du coucher du soleil) dans quelques instants et puis euh on continue à discuter ensemble si vous voulez bien $\uparrow$

(E. 2, N. 2, P. 2, L. 122-125)

Dans le premier passage, elle ajoute une formule d'invocation à Dieu, très répandue dans les pratiques langagières des locuteurs algériens. Il s'agit de demander à Dieu d'accepter la prière des fidèles avec l'expression «allah yekbel». Dans le deuxième extrait, l'animatrice passe du français à l'arabe pour annoncer l'appel à la prière. Elle s'excuse auprès de l'auditrice pour l'interruption du programme et lui propose de poursuivre la discussion juste après.

C'est ce que souligne Gardner-Chloros (1985) en constatant que les schémas linguistiques clairement identifiables correspondent à un ensemble de raisons distinctes: l'alternance lexicale par exemple, correspondrait soit à des lacunes de vocabulaire chez le locuteur, soit à une influence sociolinguistique exercée par une langue sur l'autre dans un domaine donné (tabous, coutumes, religion, idéologie...).

\subsection{Humour : jeux de langues}

Le passage d'une langue à une autre peut également avoir pour fonction l'humour, dans la mesure où les locuteurs font appel à une langue pour détendre l'atmosphère afin de rendre la conversation plus fluide et plus expressive.

Nous avons constaté que l'animateur de la troisième émission utilise toujours la même expression en arabe en s'adressant aux auditeurs qui sont au volant. L'animateur leur demande de se garer en leur racontant la blague du policier.

A : ça va $\uparrow /$ il est sur la route Karim j’imagine $\uparrow$

AU3 : oui sur la route $\uparrow /$ ça va?

A : alors il faut que tu te gares sur la bande d'arrêt d'urgence

AU3 : comme ça ça va ?

A : et c'est bon voilà $\uparrow /$ il y a le policier qui lui dit ouathaiq esyara (papiers du véhicule) (rires) bon Karim/ rapidement/ tu donnes une voix à qui ?

(E. 3, N. 1, P. 4, L. 115-120)

A : pour vue que ça dure (rires) bon dis-moi Kaci/ tu appelles de quelle région ?

AU7 : moi je suis de Tilimli/ sur la route et au volant

$\mathrm{A}:$ attention attention/ à Tilimli il y a beaucoup de policiers hein

AU7 : attention

A : attention

AU7 : il y a les flics qui rôdent

A : les flics qui rôdent/ qui surveillent (rires) et qui vous disent ouathaiq esyara sayidi (papiers du véhicule monsieur)// bon dis-moi/ soixante-dix quatre-vingts quatrevingt-dix ? pour gagner une puce

(E. 3, N. 3, P. 10, L. 331-339)

Dans ces deux extraits, les deux auditeurs appellent en conduisant et l'animateur leur conseille de se garer et de faire attention au policier qui demande les papiers du véhicule. Il fait de l'humour avec cette expression en alternant le français et l'arabe. 
Une autre expression en arabe caractérise également l'animateur de cette émission. Il s'agit d'une formule introductive dont se sert l'animateur pour présenter l'émission et la chaîne radiophonique.

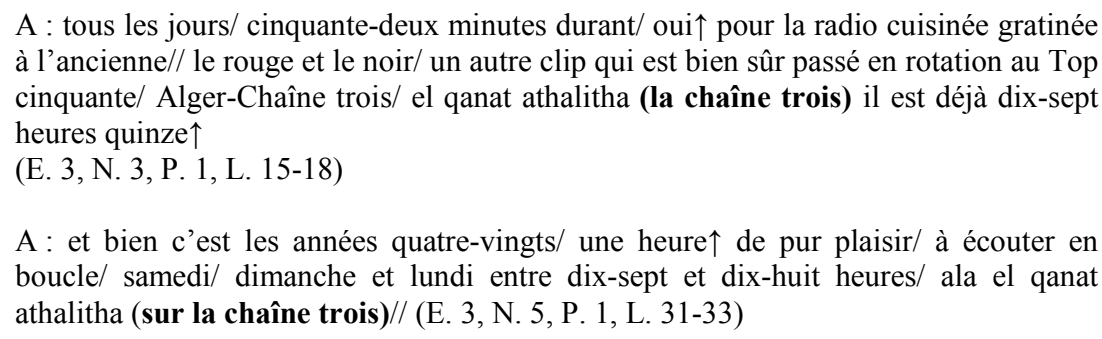

A : et bien c'est les années quatre-vingts/ une heure $\uparrow$ de pur plaisir/ à écouter en boucle/ samedi/ dimanche et lundi entre dix-sept et dix-huit heures/ ala el qanat athalitha (sur la chaîne trois)// (E. 3, N. 5, P. 1, L. 31-33)

Dans ces deux passages, l'animateur parle de l'émission et des jours de sa diffusion en faisant un jeu de langues. Dès le début du Top Ten, il fait référence à la chaîne III, une chaîne radiophonique d'expression française, en utilisant l'arabe afin de distraire les auditeurs.

Parfois, il reprend la réplique au milieu et à la fin de l'émission en rappelant aux auditeurs l'heure et le numéro de téléphone de la chaîne III.

A : mais moi je bouge pas/ je suis là samedi dimanche et lundi/ ala el qanat athalitha faqat ala el haoua oua ala el mobachir (sur la chaîne trois uniquement à l'antenne et en direct)

(E. 3, N. 1, P. 5, L. 161-163)

A : dix-sept heures trente six/ sur les ondes d'el qanat athalitha (la chaîne trois)/ aala el haoua (à l'antenne)/ aala el mobachir (en direct)/

(E. 3, N. 5, P. 11, L. 328-329)

Nous remarquons dans ces passages que l'animateur passe du français à l'arabe pour introduire sa formule habituelle accompagnée d'une autre expansion « ala el haoua oua ala el mobachir » (à l'antenne et en direct).

L'animateur réitère parfois en arabe le nom de la chaîne après l'avoir annoncée en français. Il poursuit toujours en arabe en rappelant l'heure ou le numéro de téléphone. En attestent les extraits suivants :

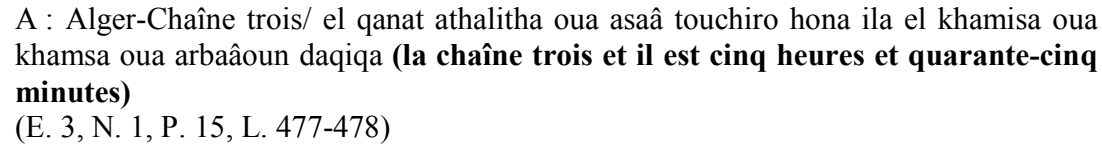

A : décollage immédiat du côté du zéro vingt et un quarante-huit euh quinze quinze/ les ondes fraîches bien sûr de la trois/ el qanat athalitha (la chaîne trois)

(E. 3, N. 3, P. 9, L, 305-306)

Dans le premier passage, l'animateur cite le nom de la chaîne en français puis en arabe et enchaîne dans cette langue. Il apporte sa touche personnelle en alternant le nom dans les deux langues. Cette tournure est très rare dans une chaîne d'expression française où le nom est souvent annoncé en français. L'intention de l'animateur est de faire rire les auditeurs.

Par ailleurs, il annonce dans le deuxième passage la fin de l'émission en donnant aux auditeurs un autre rendez-vous à la même heure et sur la même chaîne " el qanat athalitha » :

A : c'est déjà la fin dans la catastrophe/ on vous retrouve toujours avec énormément de plaisir// merci beaucoup/ j'aurai le plaisir de vous retrouver demain/ si ça vous dit 
bien sûr/ entre dix-sept et dix-huit heures/ ala el qanat athalitha (sur la chaîne trois)

(E. 3, N. 4, P. 23, L. 682-685)

Nous avons aussi recensé d'autres éléments qu'on considère comme des emprunts où l'animateur tente de détendre l'atmosphère, d'apporter un peu de gaieté à l'émission et de rendre les auditeurs plus détendus. Il s'agit d'insertion d'éléments en espagnol et en italien qui sont très fréquents dans la troisième émission, à savoir «playa » et «nostalgia ».

L'animateur fait toujours référence à un moment de détente en espagnol et toujours avec la même expression. Ainsi, il tente d'amuser les auditeurs et de les faire se détendre :
A : ah oui/ alors vacances à la playa quoi
(E. 3, N. 1, P. 9, L. 273)
A : vacances à la playa/ ok tchao
(E. 3, N. 4, P. 23, L. 667)
$\mathrm{A}$ : vacances à la playa
(E. 3, N. 5, P. 9, L. 260)

La nostalgie est également exprimée en italien. L'animateur fait référence à des chansons qui rendent les auditeurs nostalgiques, rendant ainsi cette émission plus attirante et riche en émotions :

A : après la génération des herbivores/ la génération des carnivores/ la génération des radiovores/ samedi/ dimanche et lundi/ entre dix-sept et dix-huit heures/ cinquantedeux minutes de pure nostalgia//

(E. 3, N. 4, P. 3, L. 90-92)

A : pour la dernière semaine/ la dernière ligne droite avant la fin de cette saison du Micro ondes/ on arrive à Foreigner/ «I Want To Know What Love Is »/ dernière nostalgia/

(E. 3, N. 4, P. 3, L. 101-103)

A : il suffit de pianoter/ de tapoter/ ou euh fou- euh ou former euh utiliser également le zéro vingt et un quarante-huit quinze quinze/ et vous aurez comme vous le savez/ la nostalgia/ au bout du fill/à tout de suite

(E. 3, N. 5, P. 4, L. 113-116)

Quelques éléments en arabe dans un discours en français servent aussi de jeu de langue et témoignent de l'humour de l'animateur :

A : Groove Get hein oullah ghir djat (elle est venue vraiment) (rires) ok/ Groove Get/ tu as gagné une petite puce offerte par notre notre partenaire bien sûr $\uparrow /$ euh tu laisses tes coordonnées

(E. 3, N. 2, P. 14, L. 426-428)

A : on se délecte euh en écoutant ce morceau de Robbie Williams/ vous l'avez reconnu hein $\uparrow::$ un peu de hnana (tendresse) dans ce monde de bruts/ Hanane bonsoir $\uparrow$

(E. 3, N. 2, P. 18, L. 583-584)

A : bonne boussa (bise)

(E. 3, N. 5, P. 13, L. 378)

Dans le premier extrait, l'animateur fait vraiment un jeu de mots, dans la mesure où il fait des rimes en alternant les deux segments en anglais et en arabe. En effet, "Get » de anglais rime avec "djat » de 
l'arabe (le morphème - $t$ renvoyant au pronom elle), soulignant ainsi l'arrivée du groupe "Groove Get » dans le Top Ten.

L'insertion de " hnana » et de "boussa » témoigne également de l'humour de l'animateur. Il introduit des éléments riches de sens, chaleureux et affectueux véhiculant ainsi de fortes émotions.

Nous pouvons conclure que, en réalité, différentes raisons poussent les locuteurs à faire de l'alternance et à faire appel à des emprunts dans un discours en français. Différents schémas linguistiques se recouvrent et nous avons pu recenser des facteurs externes (extralinguistiques) liés aux aspects sémantiques du discours, ainsi que des facteurs internes (linguistiques) liés aux caractéristiques du langage parlé.

Nous pouvons retenir que l'analyse de notre corpus révèle que l'alternance codique remplit diverses fonctions et que, mis à part la transmission d'un message implicite, ces changements de langue ont pour autre fonction d'imposer une stratégie, celle du sujet parlant.

En ce qui concerne le choix de langue, nous pouvons dire que la maîtrise inégale des deux langues et les sujets de conversation mais aussi les thèmes des émissions sont autant d'éléments pertinents dans le choix et le changement de langue. En effet, nous constatons que quelques stratégies de communications dépendent d'une telle émission plutôt que de telle autre et de tel locuteur plutôt que de tel autre, à savoir l'humour qui caractérise la troisième émission et précisément l'animateur qui se veut acteur principal et qui s'approprie le rôle pivot dans le déroulement de l'interaction.

Cependant, « si le schéma de Gardner-Chloros a l'avantage de regrouper d'une manière plus systématique les facteurs du code switching en des ensembles plus significatifs, elle n'a pas su éviter le problème qui consiste à ne pas distinguer clairement « facteurs » et «fonctions » du choix et de l'alternance codique » (Zongo, 2004 : 105). La proposition de Gardner-Chloros se veut à la fois une compactification et un dépassement de celles de ses prédécesseurs dont elle s'inspire. C'est ainsi qu'elle introduit un nouveau facteur, celui de la compétence des interlocuteurs (un facteur qu'elle juge antérieur même aux motivations) que Gumperz n'excluait pas comme fonction possible (il le jugeait tout simplement secondaire) (Zongo, $2004:$ 104-105).

En effet, plusieurs autres auteurs ont travaillé sur la notion de fonction des choix et de l'alternance codique, à savoir Myers-Scotton \& Ury (1977), Saville-Troike (1982), Gumperz (1982), Poplack (1988), etc.

\section{Références bibliographiques}

Ali-Bencherif, M.-Z. (2009). L'alternance codique arabe dialectal/français dans des conversations bilingues de locuteurs algériens immigrés/non-immigrés. Thèse de Doctorat, option Sciences du langage, université de Tlemcen.

Auer, P. (1999). From codeswitching via language mixing to fused lects: toward a dynamic typology of bilingual speech. International Journal of Bilingualism, 99, 309-322.

Baggioni, D. \& Robillard, D. (1997). Île Maurice : une francophonie paradoxale. Paris : L'Harmattan.

Baggioni, D. (1997). Entrelangue. In Moreau, M.-L. (éd.), Sociolinguistique - Concepts de base. Bruxelles : Mardaga, 2 édition, 140-142.

Barillot, N. (2002). Codeswitching arabe marocain/français : remarques générales et aspect prosodique. In Canut, C. / Caubet, D. (éds), Comment les langues se mélangent. Codeswitching en francophonie. Paris : L'Harmattan, 119134.

Blom, J.-P. / Gumperz. J.-J. (1972). Social meaning in linguistic structure: Code-switching in Norway. In Gumperz, J.-J. / Hymes, D. (éds), Directions in sociolinguistics. New York : Holt, Rinehart and Winston, 259-273. 
Boyd, S., Anderson, P. / Thornell, C. (1991). Patterns of incorporation of lexemes in language contact: language typology or sociolinguistics. In ESF Network on Code-Switching and Language Contact, Papers for the Symposium on code-switching in bilingual studies: theory, signifiance and perspectives. Barcelone: ESF Scientific Networks, 463-488.

Breitborde, L.B. (1983). Levels of analysis in sociolinguistic explanation: bilingual code switching, social relations and domain theory. International Journal of the Sociology of Language, 5-43.

Canut, C. (2002). Introduction. In Canut, C. / Caubet, D. (éds), Comment les langues se mélangent. Codeswitching en francophonie. Paris : L'Harmattan, 9-19.

Clyne, M. (1967). Transference and Triggering. La Haye : Nijhoff.

Deperz, C. (1999). Quelques propos métalinguistiques d'apprenants et de bilingues sur l'alternance codique. In Castellotti, V. / Moore, D. (coord.), Alternance des langues et construction de savoirs. Cahiers du français contemporain, 5, 151-166.

Duran, L. (1994). Toward a better understanding of code switching and interlanguage in bilinguality: implication for bilingual instruction. The Journal of Educational Issues of Language Minority Students, vol. 14, 69-88.

Gafaranga, M.S. (1997). Code-switching/code mixing or Kinyarwanda for all practical purposes: The base language issue. University of Lancaster, Linguistics Department.

Gal, S. (1979). Language shift. New York : Academic Press.

Gardner-Chloros, P.H. (1983). Code switching: approches principales et perspectives. La linguistique, vol. 19, fasc. 2, 21-53.

Gardner-Chloros, P.H. (1985). Choix et alternance des langues à Strasbourg. Thèse de Doctorat, Université Louis Pasteur, Strasbourg.

Gardner-Chloros, P.H. (1991). Language selection and switching in Strasbourg. Oxford : Clarendon Press.

Gumperz, J.-J. (1982). Discourse Strategies. Studies in interactional sociolinguistics. Cambridge : University Press.

Gumperz, J.-J. (1989a). Engager la conversation, introduction à la sociolinguistique interactionnelle. Paris : Editions de Minuit.

Gumperz, J.-J. (1989b). Sociolinguistique interactionnelle- Une approche interprétative. Paris : L'Harmattan.

Hamers, J.-F. / Blanc, M.H.A. (1983). Bilinguisme et bilingualité. Bruxelles : Editions Mardaga.

Haugen, E. (1989). The analysis of linguistic borrowing. Language, 26, 210-231.

Lüdi, G. \& Py, B. (2003[1986]). Etre bilingue. Berne: Peter Lang.

Lüdi, G. (1991). Les apprenants d'une L2 code-switchent-ils et, si oui, comment?. In ESF Network on CodeSwitching and Language Contact, Papers for the Symposium on code-switching in bilingual studies: theory, significance and perspectives, Barcelone : ESF Scientific Networks, 47-71.

Mabrour, A. (2007). L'alternance codique arabe/français : emplois et fonctions. Constellations francophones, 7. In http://publifarum.farum.it/ezine_articles.php?art_id=67 (Consulté le 10/07/2009).

Manaa, G. (2000). Réflexions sur les motivations des changements ou des combinaisons de langues (arabe - chaoui français) dans le discours des professeurs de français du second degré de la région de Batna. El-Tawassol, 07.

Milroy, L. / Muysken, P. (éd.). (1995). One speaker, two languages. Cross-disciplinary perspectives on codeswitching. Cambridge : University Press.

Myers-Scotton, C. / Ury, W. (1977). Bilingual Strategies: the Social Functions of Code-Switching. International Journal of the Sociology of Language, 13, 5-20.

Myers-Scotton, C. (1983). The negociation of identities in conversation: a theory of markedness and code-choice. International Journal of the Sociology of Language, 44, 115-136.

Poplack, S. (1988). Conséquences linguistiques du contact de langues : un modèle d'analyse variationniste. Langage et société, 43, 23-46. 
Queffélec, A. et al. (2002). Le français en Algérie, lexique et dynamique des langues. Bruxelles : De Boeck \& Larcier / Duculot.

Sadi, N. (2012). L'usage du français à la Chaîne III : Aspects syntactico-sémantiques. Thèse de Doctorat, option Sciences du langage, Université de Béjaia.

Saville-Troike, M. (1982). The ethnography of communication. Oxford : Blackwell.

Scotton, C.M. / Ury, W. (1977). Bilingual Strategies: the Social Functions of Code-Switching. International Journal of the Sociology of Language, 13, 5-20.

Traverso, V. (1995). Gestion des échanges dans la conversation à trois participants. In Kerbrat-Orecchioni, C. (dir.), Le trilogue. Lyon : Presses Universitaires de Lyon.

Valdès-Fallis, G. (1978). Code switching Among Bilingual Mexican-American Women: Towards an Understanding of Sex-Related Language Alternation. In Dubois, B.L. / Crouchi, M. (dir), American Minority Women in Sociolinguistic Perspective. International Journal of the Sociology of Language. 17, 73-82.

Weinreich, U. (1953). Languages in contact. La Haye : Mouton.

Zongo, B. (2004). Le parler ordinaire multilingue à Paris. Ville et alternance codique. Paris : L'Harmattan.

\footnotetext{
${ }^{1}$ Extrait d'une étude plus large de notre thèse de Doctorat intitulée L'usage du français à la Chaîne III : Aspects syntactico-sémantiques.

${ }^{2}$ Le terme « perspective » doit s'entendre dans son acception picturale, c'est-à-dire, l'art de représenter les objets sur une surface plane, de telle sorte que leur représentation coïncide avec la perception visuelle qu'on peut en avoir, compte tenu de leur position dans l'espace par rapport à l'œil de l'observateur (Zongo, 2004 : 30).

${ }^{3}$ La stratégie de communication est énoncée en termes d'intentionnalité, de facteurs et de fonctions de l'alternance codique.

${ }^{4}$ Notre corpus étant long (255 pages), nous avons opté pour une transcription orthographique plutôt qu'une transcription phonétique. De plus, «il n'existe pas aujourd'hui de système de transcription unifié. Chacun forge son système du moment que la transcription répond aux contraintes de précision, de fidélité et de lisibilité. D'une manière générale, on n'utilise pas de transcription phonétique, trop difficile à lire, mais des transcriptions orthographiques, plus ou moins standard ou adaptées. L'orthographe adaptée cherche à rendre compte de certains phénomènes de prononciation » (Traverso, $1999: 24)$.

${ }^{5}$ Nous avons condensé les extraits à l'aide de coupes présentées par [...]. L’indication des lignes avant et après chaque coupe montre la longueur des passages entre les phénomènes que nous souhaitons mettre en lumière.

${ }^{6}$ Littéralement, l'expression signifie « Dieu est gagnant ».

${ }^{7}$ Prononcé [ope] : une sorte d'interjection de la part de l'animateur.

${ }^{8}$ La traduction en français n'est pas au passif.
} 